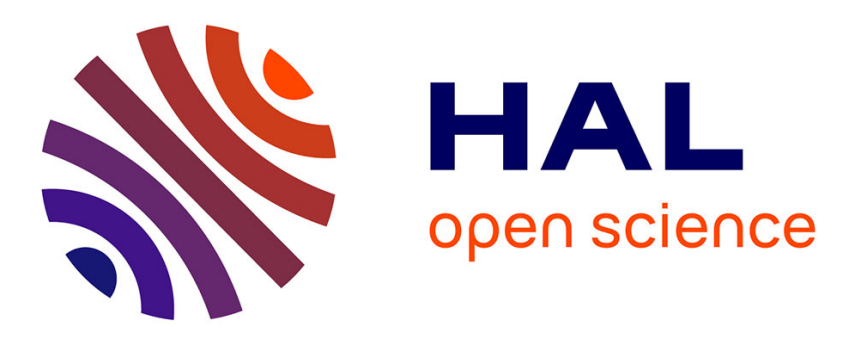

\title{
Optimization of magneto-rheological elastomers for energy harvesting applications
}

Gildas Diguet, Gaël Sebald, Masami Nakano, Mickaël Null Lallart, Jean-Yves

Cavaillé

\section{- To cite this version:}

Gildas Diguet, Gaël Sebald, Masami Nakano, Mickaël Null Lallart, Jean-Yves Cavaillé. Optimization of magneto-rheological elastomers for energy harvesting applications. Smart Materials and Structures, 2020, 29 (7), pp.075017. 10.1088/1361-665X/ab8837 . hal-02970968

\section{HAL Id: hal-02970968 \\ https://hal.science/hal-02970968}

Submitted on 22 Oct 2020

HAL is a multi-disciplinary open access archive for the deposit and dissemination of scientific research documents, whether they are published or not. The documents may come from teaching and research institutions in France or abroad, or from public or private research centers.
L'archive ouverte pluridisciplinaire HAL, est destinée au dépôt et à la diffusion de documents scientifiques de niveau recherche, publiés ou non, émanant des établissements d'enseignement et de recherche français ou étrangers, des laboratoires publics ou privés. 


\title{
Optimization of Magneto-Rheological Elastomers for energy harvesting applications
}

\author{
Gildas Diguet $^{1,2}$, Gaël Sebald ${ }^{1}$, Masami Nakano ${ }^{1,3}$, Mickaël Lallart ${ }^{1,2,4}$, and Jean-Yves \\ Cavaillé $^{1}$ \\ ${ }^{1}$ ELyTMaX UMI 3757, CNRS-Université de Lyon-Tohoku University International Joint Unit, Tohoku \\ University, Sendai, Japan \\ ${ }^{2}$ Institute of Fluid Science, Tohoku University, Sendai, Japan \\ ${ }^{3}$ New Industry Creation Hatchery Center, Tohoku University, Sendai, Japan \\ ${ }^{4}$ Univ. Lyon, INSA-Lyon, LGEF EA682, F69621 Villeurbanne, France
}

E-mail: gildas.diguet.d4@ tohoku.ac.jp

Received xxxxxx

Accepted for publication $\mathrm{xxxxxx}$

Published Xxxxxx

\section{Abstract}

Recent works demonstrated the abilities of magneto rheological elastomers (MREs) for mechanical to electrical energy conversion. This class of materials consists of composites composed of a soft elastic matrix filled by ferromagnetic particles. In this work we focus primarily on anisotropic MREs featuring particles that are non-homogenously dispersed in the matrix but form of chains of particles. Such anisotropic MRE are good candidates for energy conversion and harvesting purpose because of the coupling between high magnetization of the soft ferromagnetic particles and low elastic modulus of the matrix; thus, the anisotropic MREs can convert a mechanical cyclic deformation into electric signal. In the framework of magnifying the conversion capabilities of such materials, this study reports the investigation of different filling factors of particles, submitted to various magnetic excitations and amplitudes of mechanical deformation. The multiscale analysis provided by this work, from local effect to global characterization, shows that optimal conditions depend on intrinsic material parameters (e.g., filling factor) as well as external conditions (mainly bias magnetic flux density and applied mechanical solicitation).

Keywords: magneto rheology, composite, effective properties, energy conversion, filling factor

\section{Introduction}

Smart materials form a class of materials used because of their (multi-)coupled properties, as magnetic/mechanical responses for magnetorheological behavior. They can be used as sensors or actuators for instance. Magneto Rheological Elastomers (MREs) are composites that belong to the smart material class as they provide coupled properties between magnetic and mechanical domains. Specifically, MREs combine high magnetization of the magnetic fillers and mechanical softness of the matrix host. Most common MREs 
consist of an elastomeric matrix embedding magnetic particles, which are often magnetically soft iron (exhibiting small hysteresis and high permeability) [1]. MREs are then becoming attractive for their magnetically tunable mechanical properties [2], where the magnetic interaction between particles directly affects the composite mechanical modulus [3], bridging the gap for low frequency, high strain applications. Their tunable stiffness therefore allows the design of damping systems for instance [1-2, 4-5]. Similarly, MREs can be deformed by applying a magnetic field [6-8].

Many elastomers are obtained by chemical reaction at temperature higher than room temperature: this step is known as "curing". Such composites have then the possibility to be manufactured as anisotropic or isotropic materials. The former case is obtained by applying a constant magnetic field during the curing of the elastomer [1], the distribution of the particles inside the composite changes, while without magnetic field, the particles are randomly dispersed in the matrix. The application of such a field yields the formation of elongated particle-structures strongly oriented (called hereafter chains) along the direction of the magnetic field. This anisotropy is expected to drastically increase the effect of magnetic field on mechanical properties [9], magnetic permeability [10], magnetic field-induced deformation [11], conductivities [1213], etc. Because of the strong coupling between magnetic and mechanical properties, these materials open new applications, such as magnetically tuned dampers [14], or provide new alternatives to existing devices like sensors and/or actuators [15].

Recently [16-18], MREs were demonstrated to be effective materials for mechanical to magnetic (and then possibly electrical) energy conversion. In such applications, these materials are composed of a soft elastic silicone rubber filled with carbonyl iron particles, and present anisotropy due to the alignment of the magnetic particles during curing (through the application of a bias magnetic field). Anisotropic MREs, once placed in the air-gap of an electromagnet used to apply a controlled constant static magnetic flux density $B_{0}$, were experiencing a varying mechanical shear strain, perpendicularly to the magnetic anisotropy. Such relative displacement of the particles is known to modify their interactions, which in turn changes the MRE shear modulus $G$ under magnetic field $B$, defined as: $\Delta G(B) / G(B=0)=[G(B \neq 0)$ $G(B=0)] / G(B=0)$; this ratio is the so-called "MR effect" [3, $14,19]$. However, in the framework of energy harvesting, the shear strain $(\gamma(t))$ can be used as the way to modify the particle magnetic states and therefore to induce a magnetic flux density fluctuation $\Delta B(\gamma(t), t)=[B(\gamma \neq 0)-B(\gamma=0)]$ of the whole magnetic circuit. This change of flux is then converted into an electrical signal $V(t)$ through a "search" coil.

A previous study [16] has investigated the effect of the matrix softness, $G(B=0)$, on the direct and converse conversion effects, $\Delta B(\gamma)$ and $\Delta G(B)$. Two composite materials were manufactured with the same filling factor: one with silicone oil to soften the composite $(G(B=0)=34 \mathrm{kPa})$ and one without oil $(G(B=0)=250 \mathrm{kPa})$. Results showed same absolute change in shear modulus $(\Delta G(B) \sim 160 \mathrm{kPa})$ for both composite, but, for different relatively one $(\Delta G(B) / G(B=0)=+470 \%$ for composite with oil while $\Delta G(B) / G(B=0)=+60 \%$ without oil). Similarly, by shearing the two MREs, a flux density change of $\Delta B(\gamma=50 \%) \sim-10 \mathrm{mT}$ was measured for both composites. This shows that $\Delta B(\gamma)$ does not depend on the softness of the matrix but on the amplitude of strain. As the latter variation $(\Delta B(\gamma))$ is aimed to be converted into an electrical signal, maximal value of $\Delta B(\gamma)$ is desired. The softness of the anisotropic MRE was demonstrated to be not crucial for the electric signal [16]. Also important in the framework of electric signal conversion, this work [16] has also pointed out others key parameters to get the best $\Delta B$ : largest strain amplitude (up to $\gamma=50 \%$ ) was leading to largest $\Delta B$ it behaves as $\Delta B \sim \gamma^{2}$. Beside, the effect of the applied field was also studied and revealed the existence of an optimal applied field around 0.2-0.3 T. An important material parameter is the anisotropy of MRE: the flux density variation $\Delta B$ produced by anisotropic MRE was larger than the one produced by isotropic MRE, meaning that the electric signal was largest by using isotropic MREs; demonstrating the interest for anisotropic MREs in this application.

However, the study [16] used a single value of filling factor $\phi \sim 24 \%$. Hence, in the present study, we propose to investigate the effect of different filling factor $\phi$. The internal structure of the MRE (size of particles chain and typical distance between the particles within the chain) are extracted from measurements, and distance between chains are then estimated. These anisotropic MREs are then subjected to magneto-mechanical characterizations for several bias flux densities $B_{0}$ and shear amplitudes $\gamma_{0}$. These measurements also provide some indication on the magnetic state of the particles, and allow validating the values of the parameters corresponding to particles magnetic saturation. The electric signal production is then presented as a function of the parameters $\phi, B_{0}$ and $\gamma_{0}$. All electrical measurements show optimal pseudo-Villari effect around $0.2 \mathrm{~T}$ of bias flux density. By extracting particles magnetization curves from the MR effect assessment, the role of the particles magnetic behavior is revealed as a key parameter for energy harvesting system because their saturation arises near to the optimal field for electric signal generation.

\section{Experimental procedure}

Composites are prepared with Silicon Rubber (SR) KE1241 combined with a curing agent CLA-9 purchased from Shin-Etsu Chemical Co. Ltd. (Tokyo, Japan). The magnetic spherical particles are Carbonyl Iron (CIP) from BASF, with average diameter of $2 \mathrm{a}=6-7 \mu \mathrm{m}$. Composites are fabricated by mixing these elements with different volume filling factors for 
each sample (Table 1). After mixing and stirring the solution, the mixture was degassed by pumping the air. The final mixture was then injected in a $2 \mathrm{~mm}$ thick, $65 \mathrm{~mm}$ long and $12 \mathrm{~mm}$ wide mold which was placed in an electromagnet applying a constant flux density of $0.3 \mathrm{~T}$, along the sample thickness: the samples were cured in such conditions, creating anisotropic MREs. Obtained sheets were then cut into $2 \mathrm{~mm}$ thick, $50 \mathrm{~mm}$ long and $12 \mathrm{~mm}$ wide samples and placed inside the device.

Table 1. Experimental samples composition

\begin{tabular}{|c|c|c|}
\hline Samples & $\begin{array}{c}\text { Carbon Iron } \\
\text { Particles volume } \\
\text { fraction }(\%)\end{array}$ & $\begin{array}{c}\text { Silicon Rubber } \\
\text { volume fraction } \\
(\%)\end{array}$ \\
\hline MRE05 & 5 & 95 \\
\hline MRE10 & 10 & 90 \\
\hline MRE15 & 15 & 85 \\
\hline MRE20 & 20 & 80 \\
\hline MRE25 & 25 & 75 \\
\hline
\end{tabular}

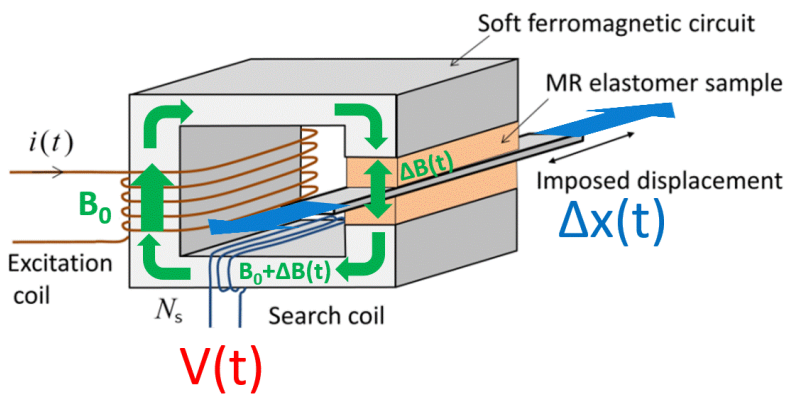

Fig.1. Schematics of the characterization device

The global device is represented in Fig.1. It is composed of a soft ferromagnetic circuit with cross section $A$ of $50 * 12 \mathrm{~mm}^{2}$ with a length of $200 \mathrm{~mm}$ plus a $5 \mathrm{~mm}$ air gap. Around this soft ferromagnetic material, two coils are winded: one excitation coil $\left(N_{\mathrm{p}}=1560\right.$ turns $)$ in order to apply a controllable static magnetic flux density $B_{0}$, and another one $\left(N_{\mathrm{S}}=300\right.$ turns) to pick up the electrical signal produced by the variation of magnetic flux in the circuit. Magnetic flux was calibrated as described in [16]: the excitation coil was supplied by a constant current and induction was determined by a fluxmeter (FM-3001, Denshijiki Industry Co.). Relationship between current and induction was calibrated for each sample. The circuit gap is filled with two samples separated by a $1 \mathrm{~mm}$ in thickness steel blade. The steel blade is magnetized as the magnetic field is applied in the system. This, actually helps to canalize the magnetic flux in the whole circuit. If the blade wasn't magnetic, the flux circuit would exhibit larger leakage, and the field experienced by the MREs would be slightly lower.
Each samples are submitted to transverse shear strain applied through a displacement $\Delta x$ of the steel blade. The displacement amplitudes were set to $\Delta x=0.2,0.4,0.6,0.8$ and $1 \mathrm{~mm}$ corresponding to respective maximal amplitude of shear strain of $\gamma_{0}=10,20,30,40$ and $50 \%$ of the two $2 \mathrm{~mm}$ thick samples. This imposed strain has a sinewave shape with a frequency $f$ of $1 \mathrm{~Hz}$ :

$$
\gamma(t)=\gamma_{0} \sin (2 \pi f t)
$$

Displacement, stress and voltage were recorded every millisecond for two periods, by means of a strain gauge, a stress cell force and an oscilloscope connected to the search coil. Applied flux density was changed from 0 to $0.7 \mathrm{~T}$ (except for MRE05 and MRE10, where the maximal value was $0.6 \mathrm{~T}$ ). From these measurements, the shear modulus was determined for different applied fields and strain amplitudes.

The permeability of MRE samples was measured using a Magnetic Permeability Meter Ferromaster from Stefan Mayer Instruments. This instrument is capable of measuring the relative permeability in the range of $\mu_{\mathrm{r}}=1$ to 2 with an accuracy of 0.001 .

\section{Results and discussion}

\subsection{Anisotropic composite characterization}

The time-dependent shear strain induces a time-varying MRE magnetic permeability through the interaction of the mechanical solicitation of the composite with the magnetic fillers. More precisely, the magnetic permeability of such composite, $\mu_{\mathrm{MRE}}=\mu_{0} \mu_{\mathrm{r}}$ (with $\mu_{0}$ the vacuum permeability $\mu_{0}$ $=4 \pi 10^{-7} \mathrm{H} / \mathrm{m}$ and $\mu_{r}$ the relative permeability), strongly depends on the inter-particle distance, which is in turn related to the shear strain amplitude. In anisotropic composites with chain-like structures, the inter-particle distance is much smaller within the chains than between neighboring chains. Hence, interactions within the chains are expected to dominate the magnetization process of the ferromagnetic particles in the anisotropic MRE. The relative permeability component taken along the chain axis is expressed, at rest $(\gamma=0)$, by [18]:

$$
\begin{aligned}
\mu_{r}(\phi, \gamma=0)= & 1+\frac{3 \phi}{1-\left(\phi+4 S\left(\frac{a}{d}\right)^{3}\right)} \\
& =1+\frac{3 \phi}{1-\left(\phi+4 S(2 h)^{-3}\right)}
\end{aligned}
$$

where $\phi$ is the composite filling factor, $d$ is the initial interparticle distance inside the chain, $a$ is the particle radius and $S=1.202$ refers to a coefficient for summation over an infinite perfectly aligned particles chain [18] and $h=d / 2 a$ is the ratio between the inter-particle distance within a chain to the particle diameter. Setting a structure constant as $2 D=$ $4 S(a / d)^{3}$, for $D=0$, equation (2) becomes the well-known 
expression for a dilute random dispersion of ferromagnetic particles in a non-magnetic matrix, namely the Maxwell Garnett (MG) permeability $\mu_{\mathrm{r}}(\phi)=1+3 \phi /(1-\phi)$ [19] which is independent from the strain. This therefore highlights the benefit in using anisotropic MREs for coupling mechanical and magnetic domains, as such configuration allows breaking the randomness.

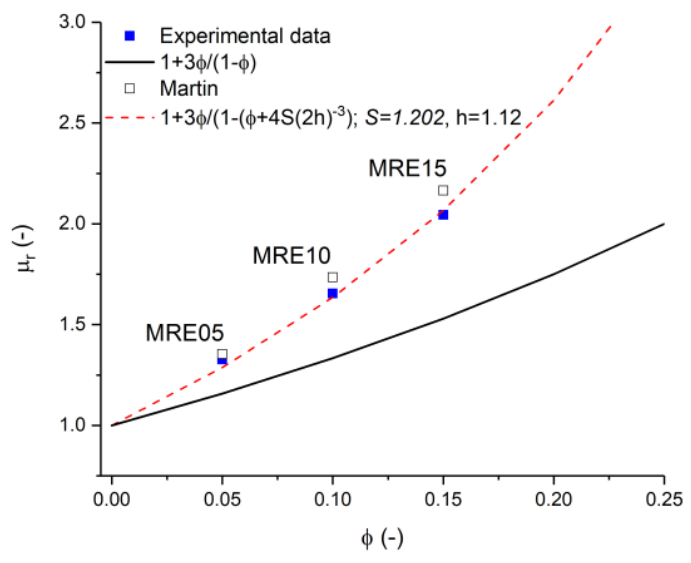

Fig.2. Experimental relative permeability of anisotropic composites versus filling factor, theoretical permeability using equation (2) with $h=1.12$, theoretical Maxwell Garnett for dilute isotropic composite [19] and numerical anisotropic composite from Martin [20]

Such unstrained permeabilities were measured for different composite filling factors $\phi=5,10$ and $15 \%$ as $\mu_{\mathrm{r}}=1.33,1.65$ and 2.04 as shown in Fig.2. Higher filling factor were out of range of the permeability meter. We also added, the corresponding predicted isotropic MG permeabilities as $\mu_{\mathrm{r}}=$ 1.16, 1.33 and 1.52 to demonstrate the higher permeability offered by a structured MRE from the measurements (this work) or using the anisotropic relative permeability numerically calculated by Martin as $\mu_{\mathrm{r}}(\phi)=1+3 \phi /\{1-(\phi$ $2 \Psi(\phi))\}[20]$ which is also independent from the strain but has a structure term $2 \Psi(\phi)$ which vary with the filling factor. Calculated Martin permeabilities are $\mu_{\mathrm{r}}=1.35,1.73$ and 2.16 for the filling factors $\phi=5,10$ and $15 \%$ respectively.

From these measurements and using equation (2), it is possible to extract the initial ratio of inter-particle distance to particle radius, $h$ for each composite as:

$$
\mathrm{h}=\frac{1}{2}\left[\frac{-4 S}{\frac{3 \phi}{\mu_{r}(\phi, \gamma=0)-1}-1+\phi}\right]^{1 / 3}
$$

And the results are inserted in Table 2. Such results show that the average distance between particles in the chains is very small as expected. As an example, we plotted the permeability versus filling factor curve using equation (2) with $h=1.12$.
From the calculated $\mathrm{h}$ values in Table 2, we can estimate the inter-chain distance by defining a unit cell that contains a single particle with the dimensions constrained by the filling factor $\phi$. Assuming this unit cell height is $d$ while width and depth are $w$, as seen in the inset of Fig. 3, then the following relationship holds:

$$
\phi=\frac{\frac{4}{3} \pi a^{3}}{d w^{2}}
$$

By injecting the measured $d=2 h a$, then the inter chain distance $w$ can be estimated as:

$$
\frac{w}{a}=\sqrt{\frac{4 \pi}{6 h} \frac{1}{\phi}}
$$

In this case, we used $d=2.14 \mathrm{a}, 2.21 \mathrm{a}$ and $2.25 \mathrm{a}$ then we obtain $w=6.26 a, 4.35 a$ and $3.52 a$ for $\phi=5 \%, 10 \%$ and $15 \%$ respectively. Similar tendency was found using the permeabilities of Martin and equation (2) and (5) for corresponding filling factor; extracted data are added in Fig.3. This confirms that the average distance between particles inside the chain is much smaller than the distance between chains as initially supposed. Since the dipole-dipole interaction behaves as the inverse of the cubic distance, the chains can be considered almost isolated. Calculated unit cell dimensions are plotted in Fig.3, as a function of the filling factor. It can be also noted that the height remains smaller compared to the width and depth.

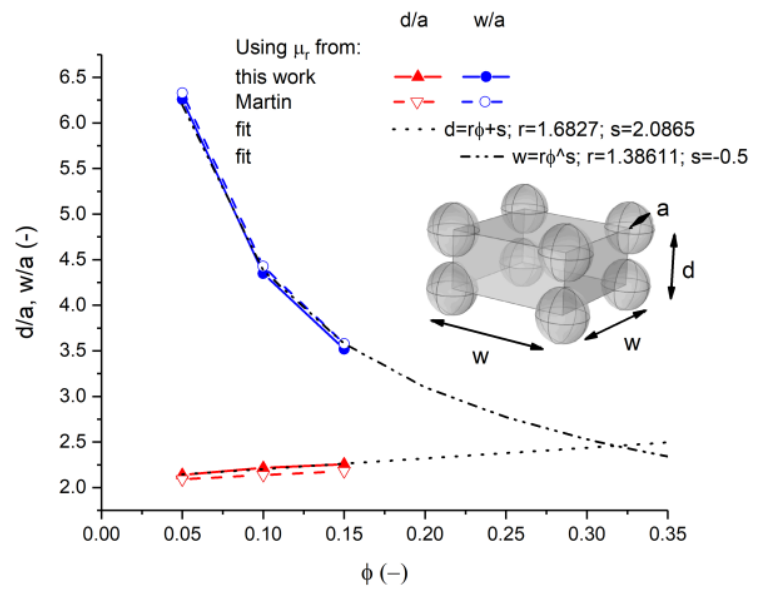

Fig.3. Distance $d / a$ with the estimation of the distance between chain $w / a$ from equations (3) and (5).

As it can be seen from Fig.3, the anisotropy degree is obviously decreasing with increasing the filling factor. Assuming that the distance $d$ is linearly increasing with $\phi$ while $w$ is decreasing as $w \sim \phi^{-1 / 2}$ (as in equation 5), an intersection can be found at a filling factor of $\phi \sim 0.32-0.33$, 
which is close to the observed value of 0.33 by magnetic characterization [21] or by image analysis [22]. Furthermore, we can notice that this limit occurred before the theoretical maximal packing $\phi=\pi / 6 \sim 0.52$ of the simple cubic unit cell with $d=w=2 a$ when particles are in contact.

Table 2. Parameters $h$ of the fitted curves in Fig.2 using equation (3), the corresponding $d$ and $w$ using equation (5) for each sample, and parameters $q$ of the fitted curves in Fig.5 using equation (7) for each sample.

\begin{tabular}{|c|c|c|c|c|c|}
\hline & MRE05 & MRE10 & MRE15 & MRE20 & MRE25 \\
\hline$h(\mathrm{a})$ & 1.07 & 1.11 & 1.13 & - & - \\
\hline$d(\mathrm{a})$ & 2.14 & 2.21 & 2.25 & & \\
\hline$w(\mathrm{a})$ & 6.26 & 4.35 & 3.52 & & \\
\hline$q(-)$ & 8.82 & 6.82 & 6.57 & 6.02 & 6.87 \\
\hline
\end{tabular}

The mechanical reinforcement of the composites with different filling factor is presented in Fig. 4 depicting the stress-strain curves obtained at $\gamma_{0}=10 \%$ without magnetic field $\left(B_{0}=0.0 \mathrm{~T}\right)$. The increase of the inclination of these elliptic-shaped curves clearly shows that the samples are getting stiffer as the filling factor increases. By assuming that each stress-strain curves ( $\tau$ versus $\gamma$ ) is a perfect ellipse, we can quantify the composite stiffness. The measured stress has a sine form as:

$$
\tau=\tau_{0} \sin (2 \pi f t+\delta)
$$

where $\tau_{0}$ is the maximal amplitude of measured stress and $\delta$ is a phase difference between stress and strain.

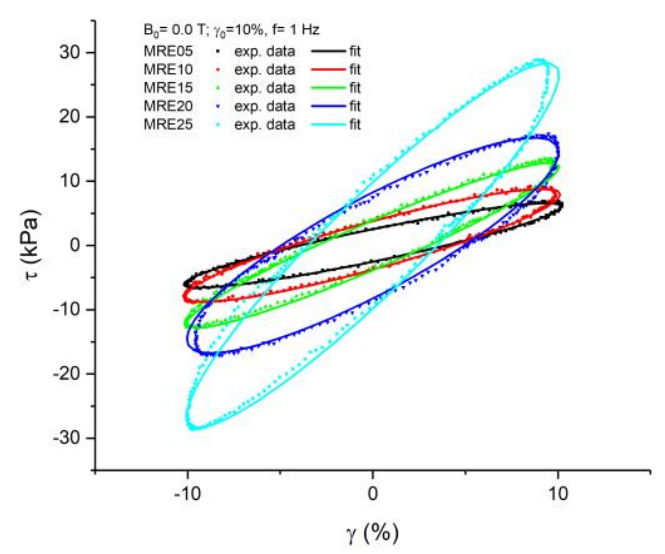

Fig.4. Stress-strain curves obtained at $\gamma_{0}=10 \%$ for each sample without magnetic field $B_{0}=0.0 \mathrm{~T}$.

We used a classical linear viscoelastic model [16, 23] to fit these curves in order to estimate separately the elastic modulus $G^{\prime}\left(\phi, B_{0}\right)$ and loss modulus $G^{\prime}\left(\phi, B_{0}\right)$, as:

$$
G^{\prime}=\frac{\tau_{0}}{\gamma_{0}} \cos (\delta) ; G^{\prime \prime}=\frac{\tau_{0}}{\gamma_{0}} \sin (\delta)
$$

In general, $G$ ' is function of frequency $f$, strain magnitude $\gamma_{0}$, filling factor $\phi$, and bias flux density $B_{0}$ where dependency can be modeled by power-law functions. In Fig.5, extracted values of $G^{\prime}$ are then plotted versus the filling factor $\phi$, for $f=1 \mathrm{~Hz}, \gamma_{0}=10 \%$ and $B_{0}=0.0 \mathrm{~T}$. Because these anisotropic composites contain long chains of particles, we use the model of Guth [24] for random dispersion of elongated particles (with aspect ratio $q>1$ ) in a matrix. This model is expressed as:

$$
G^{\prime}(\phi)=G_{0}^{\prime}\left(1+0.67 q \phi+1.62 q^{2} \phi^{2}\right)
$$

where $G_{0}$ is the pure matrix shear modulus and $q$ the chain of particles aspect ratio. Example of such reinforcement using $G^{\prime}{ }_{0}=38.5 \mathrm{kPa}$ and $q=6.6$ is plotted in Fig.5. The Guth and Gold mechanical modulus for isotropic materials [25] considering the random dispersion of spherical particles in a matrix and expressed as $G^{\prime}=G_{0}^{\prime}\left(1+2.5 \phi+14.2 \phi^{2}\right)$, is also plotted using the same shear modulus $\left(G^{\prime}{ }_{0}=38.5 \mathrm{kPa}\right)$ in Fig. 5 to highlight the anisotropy of these composites. Flandin [26] has used this equation (8) for carbon black-filled ethyleneoctene elastomer composites with filling factor ranging from 0 up to $25 \%$ found a value of $q=4.85$ [26] which validated our approach. So, keeping the value of $G_{0}{ }_{0}$ fixed, more precise evaluations of $q$ are extracted for each MRE and results are presented in Table 2 with values are ranging between 6 to 8 .

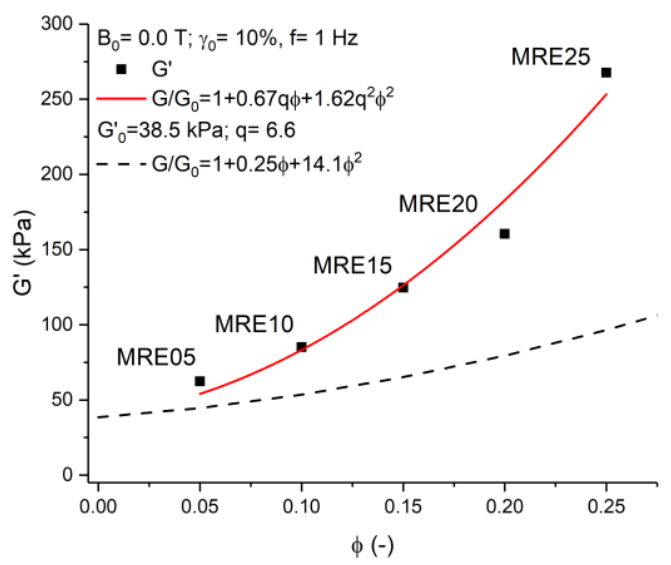

Fig.5. Storage shear modulus curves obtained without magnetic field $B_{0}$ at $\gamma_{0}=10 \%$ versus sample filling factor. The solid red fitting curve corresponds to the Guth model [24] of random dispersion of particles in a matrix with aspect ratio $q=6.6$ for mechanical modulus and dash line stands for Guth and Gold [25] model for mechanical modulus of isotropic composites. 
The experimental moduli are indeed larger than isotropic composites moduli for the same filling factor. From the nonstrained permeability measurements and the zero field mechanical characterization, the anisotropic composite is schematically pictured as a random dispersion of chains where the intra-chain distance between particles, is nearly 2.24 times the particles radius (the distance between particles edge is 0.24 times the particles radius) and with an approximate chain aspect ratio of 7 .

\subsection{Magneto mechanical characterization}

The application of a magnetic field to the composite induces a magnetic moment in each ferromagnetic particle and each of these particles then interacts each other. The magnetization of the particles creates a body force inside the volume of the whole composite. This force is known to be responsible for the apparent magnetostriction if the sample is free to deform, otherwise this force results in an increase in the shear modulus. In Fig. 6, the stress-strain curves are plotted for a small applied shear strain $\left(\gamma_{0}=10 \%\right)$ and for different magnetic fields. Mechanical reinforcement is shown to be stronger with increasing applied field.

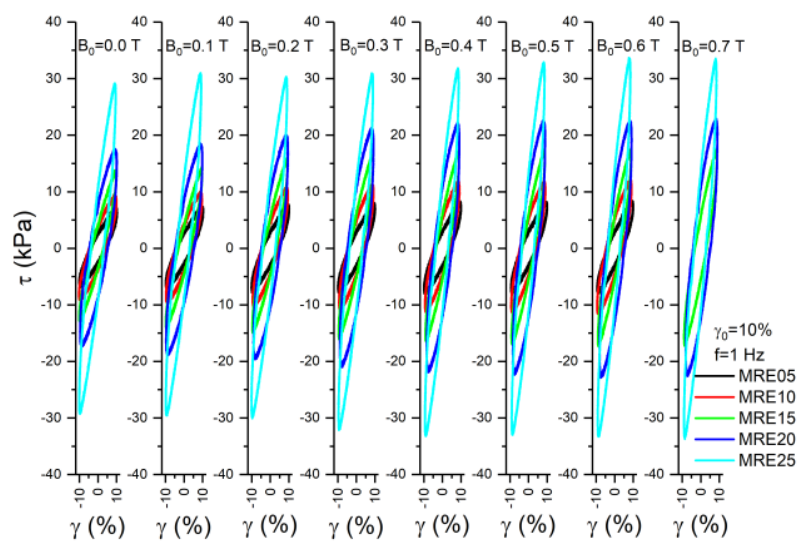

Fig.6. Stress-strain curves obtained at $\gamma_{0}=10 \%$ for each sample at given value of magnetic field.

As an example, MRE25 sample reaches a stress value around $29 \mathrm{kPa}$ for $B_{0}=0.0 \mathrm{~T}$ and $34 \mathrm{kPa}$ for $B_{0}=0.7 \mathrm{~T}$, respectively. In fact, the shear modulus is usually split into a non-magnetic part and a magnetic contribution as:

$$
G^{\prime}\left(\phi, B_{0}\right)=G^{\prime}\left(\phi, B_{0}=0\right)+\Delta G^{\prime}\left(\phi, B_{0}\right)
$$

The first term, $G^{\prime}\left(\phi, B_{0}=0.0 \mathrm{~T}\right)$, corresponds to the mechanical behavior of the matrix reinforced by the particles, as plotted in Fig. 5, while $\Delta G^{\prime}\left(\phi, B_{0}\right)$ accounts for the magnetic interactions of the fillers and is referred as MR effect. The extracted values of each anisotropic MRE $\Delta G^{\prime}\left(\phi, B_{0}\right)$ is plotted versus applied field in Fig. 7.

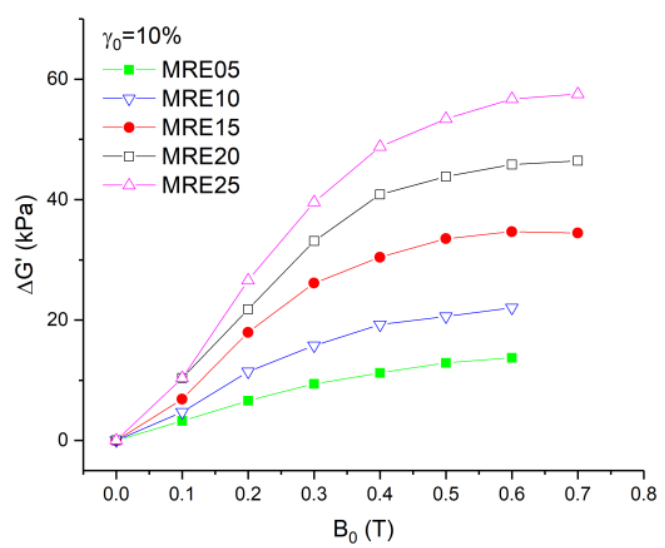

Fig.7. $\Delta G^{\prime}\left(\phi, B_{0}\right)$ dependence with applied flux density.

For large enough magnetic fields, the value of $\Delta G^{\prime}\left(\phi, B_{0}\right)$ tends to a constant value $\Delta G{ }^{\prime}$ sat $\left(\phi, B_{0}\right)$. The larger filling factor of the composite, the larger $\Delta G{ }^{\prime}$ sat $\left(\phi, B_{0}\right)$. Jolly [3] derived the change of shear modulus $\Delta G^{\prime}\left(\phi, B_{0}\right)$ from the calculation of the magnetic force between two magnetized particles, which are experiencing a small pure shear strain $\left(\gamma_{0}<10 \%\right)$, and proposed the following relationship:

$$
\Delta G^{\prime}\left(\phi, B_{0}\right)=\frac{\phi M_{p}^{2}}{2 \mu_{0} h^{3}}
$$

where $\phi$ is the composite filling factor, $h=d / 2 a$ with $d$ the initial inter-particle distance inside the column and $a$ the particle radius, $M_{\mathrm{p}}$ is the magnetization state of a particle (magnetization of each particle is assumed to be identical because of the very small shear). At sufficiently large $B_{0}$, the magnetization state of particles is reaching the saturation $M_{\mathrm{p}} \sim$ $M_{\text {sat_p }}\left(\mu_{0} M_{\text {sat_p }}=2.14 \mathrm{~T}\right)$, which in turn explains the mechanical property saturation $\Delta G_{\text {sat. }}$. From the experimental conditions, the largest applied field for all samples is $B_{0}=0.6$ $\mathrm{T}$, and we assumed that the values $\Delta G^{\prime}\left(B_{0}=0.6 \mathrm{~T}\right) \sim \Delta G^{\prime}$ sat. Then, from this relationship, we can extract the magnetization state of particles as: $M_{\mathrm{p}}\left(\phi, B_{0}\right) / M_{\text {sat_p }}=\left(\Delta G^{\prime}\left(\phi, B_{0}\right) / \Delta G_{\text {sat }}\right)^{1 / 2}$. Resulting curve is plotted in Fig.8. 


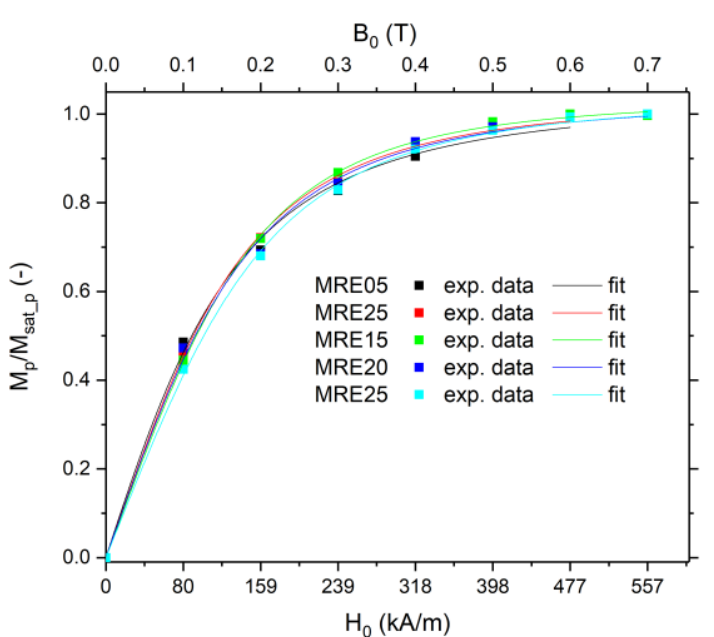

Fig.8. Relative magnetization of the particles at different value of magnetic field.

A satisfactory fit of a non-linear magnetization curve is given by $\mathrm{f}(x)=c x /(1+[c x / p] \alpha)^{1 / \alpha}$ where $\mathrm{f}(x) \sim c x$ for $x<<1$ and $\mathrm{f}(x) \sim p$ for $x>>1$, where $c$ represents here the initial magnetic susceptibility $(c \sim \chi), p$ the saturation state of the magnetization $\left(p \sim M_{\text {sat_p }}\right)$ and $\alpha$ is a curvature parameter. The magnetization of a particle versus applied magnetic field is then written as:

$$
M_{p}\left(H_{0}\right)=\frac{\chi H_{0}}{\left(1+\left[\frac{\chi H_{0}}{M_{\text {sat_p }}}\right]^{\alpha}\right)^{1 / \alpha}}
$$

This figure reveals two important points. Firstly, the particles are clearly reaching their magnetic saturation within the range of experimental applied field. Moreover, it appears that the linear behavior of the magnetic particles is limited to a relatively small range of applied field. Such a linear behavior is characterized by the magnetic susceptibility of materials. Then, the extracted susceptibilities using fitted curves in Fig.8 are presented in Table 3.

Table 3. Parameters of the fitted curves in Fig.8, using equation (8); $M_{\text {sat_p }}=1.7610^{6} \mathrm{~A} / \mathrm{m}$ for all iron particles.

\begin{tabular}{|c|c|c|c|c|c|}
\hline & MRE05 & MRE10 & MRE15 & MRE20 & MRE25 \\
\hline$\chi(-)$ & 11.35 & 10.69 & 10.01 & 10.47 & 9.32 \\
\hline$\alpha(-)$ & 1.83 & 2.07 & 2.30 & 2.06 & 2.23 \\
\hline $\begin{array}{c}\mu_{0} H_{\text {sat_p }} \\
(\mathrm{T})\end{array}$ & 0.19 & 0.20 & 0.22 & 0.21 & 0.23 \\
\hline
\end{tabular}

The extracted particle susceptibilities where around 10, which is larger than the usual isolated spherical ferromagnetic particle susceptibility of $\chi \sim 3$, which corresponds to the second notable point. Interestingly, the chains of particles were modeled [17] as segment having a susceptibility of 9.09 for a MRE with $\phi=26.3 \%$, which shows good agreement with our experimental data. The apparent susceptibility of an isolated iron particle is $\chi \sim \chi_{\mathrm{Fe}} /\left(N \chi_{\mathrm{Fe}}+1\right)$ where iron has a typical magnetic susceptibility $\chi_{\mathrm{Fe}} \sim 1000$. As the demagnetizing factor for a sphere is $N=1 / 3$, the apparent susceptibility is $\chi \sim 3$. Using these expressions, we can confirm that these chains of particles behave as elongated particles $(N<1 / 3)$ yielding the enhanced susceptibility. From the susceptibilities listed in Table 3, we can obtain effective demagnetizing factor of $N \sim 1 / \chi \sim 0.1$ which corresponds to an aspect ratio around $q \sim 4$ [27], which is a little smaller than extracted values from Fig. $5(q=6.6)$. This aspect ratio value of 4 is found by the magnetic part of the shear modulus whereas the value 6.6 is found by the non-magnetic part of the shear modulus. Both methods are nonetheless pointing to the same order of on the elongated aspect ratio of the chains.

As chains exhibit larger susceptibility than isolated particles, they reach a magnetic saturation at lower applied magnetic field. A useful parameter to quantify the saturation effect is the saturating field, defined as $H_{\text {sat } \_ \text {p }}=M_{\text {sat_p }} / \chi$. Then $\mu_{0} H_{\text {sat_p }}=4 \pi 10^{-7} \times 1.7610^{6} / 3 \sim 0.7 \mathrm{~T}$ for the isolated particle. Table 3 provides the values for the tested MREs. Values are mostly around $0.2 \mathrm{~T}$ which indicates that the MRE particles reach a magnetic saturation at lower field than isolated ones. Increasing the filling factor in the MREs seems to lower their particles susceptibilities, hence increasing the saturating field

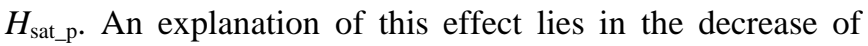
anisotropy with the increase of the filling factor, as explained in Section 3.1. Moreover, clusters of particle chains appears with the increase of the filling factor [22, 28-29].

\subsection{Electrical signal characterization}

One major application of the MRE in the framework of this investigation is the electrical signal production, for sensing or energy harvesting purposes for instance. In this analysis, different experimental parameters are considered for optimizing such targets. When the (anisotropic) MREs are submitted to a shear strain applied perpendicularly to the columnar structure, their permeabilities change, due to the rearrangement of the magnetic particles. That change of permeability induces a change of flux flowing in the magnetic circuit, producing in turn an electrical signal in the pick-up coil, as shown in Fig. 9 for MRE20, submitted to a to shear strain of $\gamma_{0}=30 \%$, with $B_{0}=0.3 \mathrm{~T}$. Considering an applied strain with a period of $1 \mathrm{~s}(f=1 \mathrm{~Hz})$, the voltage signal exhibits a period of $0.5 \mathrm{~s}$ so that the voltage frequency is twice that of the applied strain. 


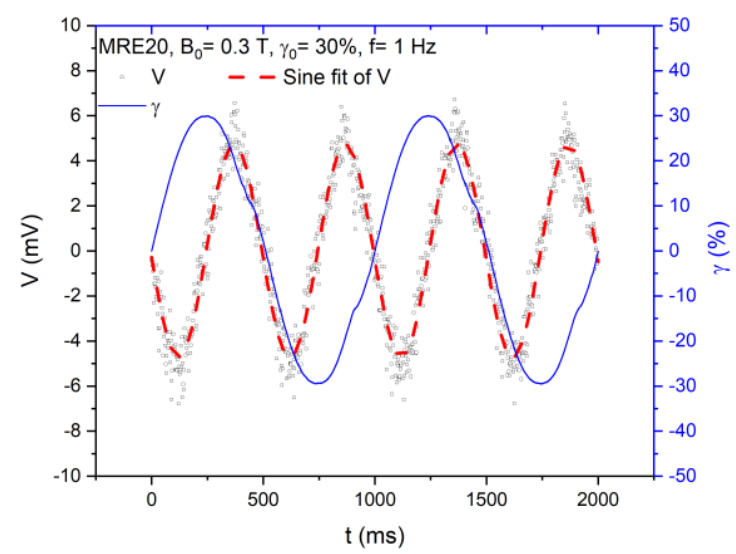

Fig.9. Voltage across the pick-up coil versus time considering MRE20 sample, submitted to $\gamma_{0}=30 \%$ shear strain (solid line) with a bias flux density of $B_{0}=0.3 \mathrm{~T}$.

By fitting the electrical signal with a sinewave function, the electric signal amplitude $V$ s for each MRE can be determined. Such data are obtained for various combinations of experimental conditions, namely the shear strain maximal amplitude $\left(\gamma_{0}\right)$ and the value of the applied field $\left(B_{0}\right)$. Resulting $V$ s are then plotted as a function $B_{0}$ for all MRE samples at given $\gamma_{0}$. As it can be observed from Fig. 10, the voltages behave similarly for all filling factors. As the applied strain $\gamma_{0}$ increases, the measured voltage also increases whatever the filling factor. The effect of the applied field $B_{0}$ is however more complex: as $B_{0}$ increases, $V$ s first increases, then reaches an optimal value and eventually decreases to zero. That optimal value $V \mathrm{~s}$ appears for $B_{0}=B_{\text {opt }}$. The value of $B_{\text {opt }}$ depends on the filling factor and strain maximal amplitude. For example, the $V \mathrm{~s}$ vs $B_{0}$ curves for MRE05 ( $\left.\phi=5 \%\right)$ presents an optimal value $B_{\text {opt }}=0.1 \mathrm{~T}$ for all $\gamma_{0}$. At increasing filling factor, $B_{\text {opt }}$ value changes, depending on $\gamma_{0}$. For MRE10 $(\phi$ $=10 \%), B_{\mathrm{opt}}=0.1 \mathrm{~T}$ if $\gamma_{0}<50 \%$ and $B_{\mathrm{opt}}=0.2 \mathrm{~T}$ if $\gamma_{0}=50 \%$. For MRE15 $(\phi=15 \%)$, for strain amplitude up to $\gamma_{0}=20 \%$, optimum value is $B_{\mathrm{opt}}=0.1 \mathrm{~T}$, but moves to $B_{\mathrm{opt}}=0.2 \mathrm{~T}$ for larger $\gamma_{0}$. For MRE20 $(\phi=20 \%)$ the value $B_{\mathrm{opt}}=0.1 \mathrm{~T}$ appears only for $\gamma_{0}=10 \%$ and then $B_{\mathrm{opt}}=0.2 \mathrm{~T}$ for other considered strain amplitudes. Finally, for the largest tested filling factor, MRE25 $(\phi=25 \%), B_{\mathrm{opt}}=0.2 \mathrm{~T}$, almost independently of strain amplitude.

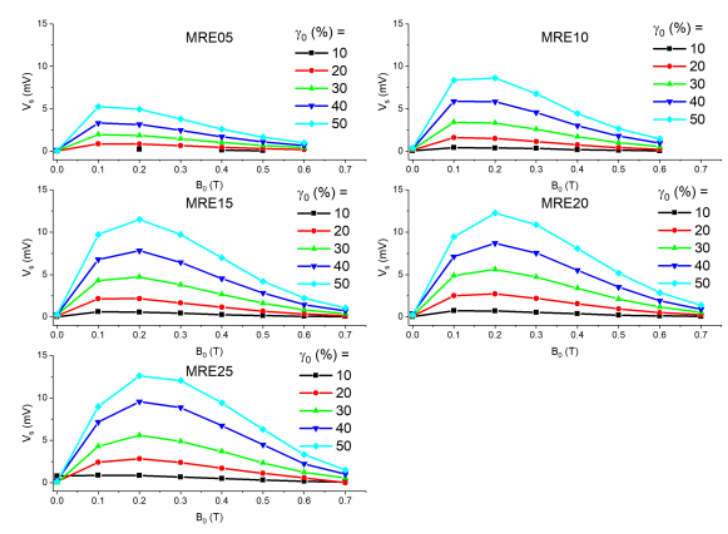

Fig.10. Amplitude of voltage $V$ s for each MRE, i.e. each filing factor, at different amplitude $\left(\gamma_{0}\right)$ and value of the applied DC field $\left(B_{0}\right)$.

It can be noted that these curves are not symmetric: the decrease is smoother than the increase. To study the effect of the material properties, the change of magnetic induction $\Delta B(t)$ is extracted from the electrical signal by integrating the Lenz law:

$$
\mathrm{V}(t)=-N_{S} A \frac{d B(t)}{d t}
$$

with the constant $N \mathrm{~s} A=0.18 \mathrm{~m}^{2}$ and removing the integration constant. The corresponding results are plotted in Fig. 11 depicting $\Delta B(t)$ versus the applied shear strain. Fig. 11(a), which illustrates the variation in flux density for different amplitudes of the strain applied to MRE25, shows that the variation in induction is quadratic with the strain. Hence, the higher the applied strain, the larger the variation of induction and therefore the larger the output voltage.

Fig. 11(b), showing $\Delta B$ versus $\gamma$ for different amplitudes of the applied field for MRE25, demonstrates that $\Delta B$ increases with $B_{0}$ up to $0.2 \mathrm{~T}$. The $\Delta B$ curves are then smaller. This is consistent with the plot of $V \mathrm{~s}$ versus $B_{0}$ for the MRE25 at $\gamma_{0}=$ $50 \%$, exhibiting an optimal field of $B_{\text {opt }}=0.2 \mathrm{~T}$ as seen in Fig.10. We can also notice the close relationship between $B_{\text {opt }}$ and the saturation field $H_{\text {sat_p }}$ as measured in Fig. 8, (see also Table 2). 


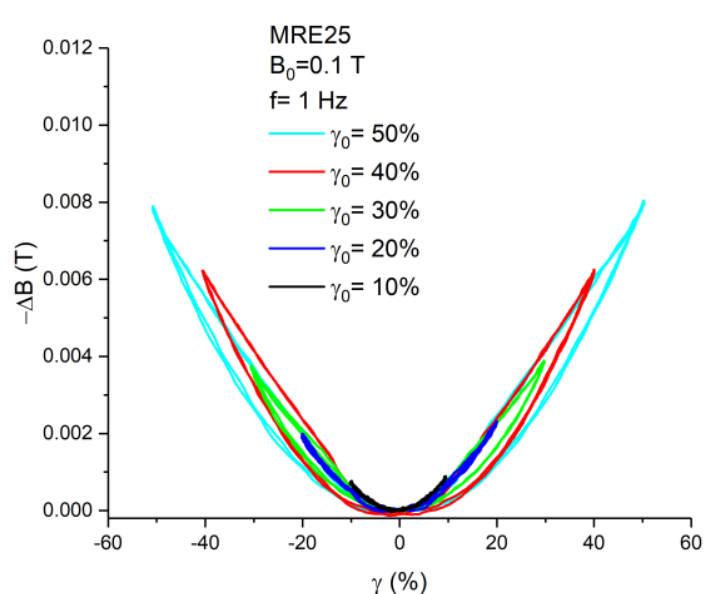

(a)

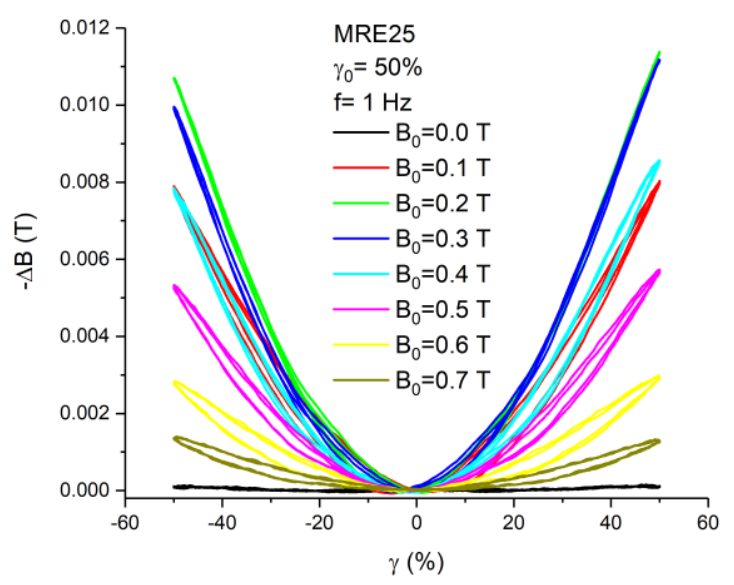

(b)

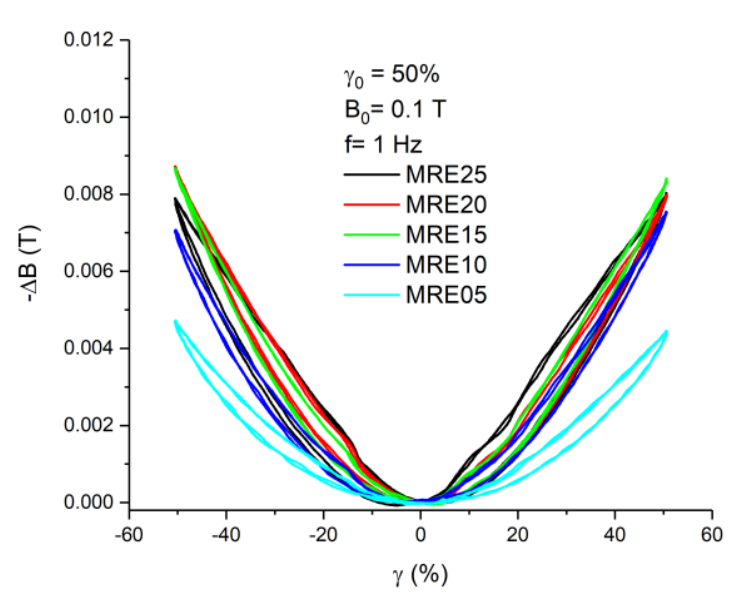

(c)

Fig.11. Change of induction versus the shear deformation, for different a) amplitudes of the shear deformation amplitude $\gamma_{0}, \mathrm{~b}$ ) applied field $B_{0}$ and c) filling factor $\phi$.
Lastly, in Fig.11.c, the evolution of $\Delta B$ versus $\gamma$ is presented for different filling factors (and thus samples), considering $\gamma_{0}$ $=50 \%$ and $B_{0}=0.1 \mathrm{~T}$ (which is close to $B_{\text {opt }}$ for all MRE samples). For increasing filling factor, $\Delta B$ tends to a similar value. For MRE05 $(\phi=5 \%)$ the absolute value of $\Delta B$ at $\gamma=$ $50 \%$ is around $5 \mathrm{mT}$ whereas larger filling factors provide approximate values of $\Delta B$ at $\gamma=50 \%$ of $7 \mathrm{mT}, 8 \mathrm{mT}, 9 \mathrm{mT}$ and $9 \mathrm{mT}$ for $\phi=10 \%, 15 \%, 20 \%$ and $25 \%$ respectively. This is again consistent with the voltage amplitudes obtained with $\gamma_{0}=50 \%$ and $B_{0}=0.1 \mathrm{~T}(V \mathrm{~s}=5.2 \mathrm{mV}, 8.4 \mathrm{mV}, 9.7 \mathrm{mV}, 9.4$ $\mathrm{mV}$ and $9 \mathrm{mV}$ for $\phi=5 \%, 10 \%, 15 \%, 20 \%$ and $25 \%$ respectively; see Fig.10). This experimental data shows a flat produced voltage with larger filling factor within our experimental test range. Such an effect can be attributed to segment-segment interaction (which is negligible for low filling factors) as described above.

\subsection{Magneto Mechanical energy conversion}

Previous investigations highlighted that larger shear provided better output voltage, and that an optimal field around $0.1 \mathrm{~T}-0.2 \mathrm{~T}$ also provides the best electric signal while larger filling factors did not provide significant improvement after a critical value. Such effects can also be considered from an energy point of view. To do this, we establish a definition of efficiency and calculate the values associated with the tested parameters.

Efficiency is here calculated by the ratio of the energy output divided by the energy inputs during a cycle of shear strain. The considered energy input is the mechanical energy to strain the MRE, at given applied magnetic field. The energy output is the change of magnetic energy due to this shear. In this way, we mainly consider what is happening in the MRE material itself. More precisely, the way the bias field is created, the way actuators apply strain or the pick-up coil conversion efficiency are out of the scope of the analysis. These are device issues that can be optimized in future works.

The change of magnetic density energy $E_{\mathrm{mag}}$ due to the shear $\gamma$ for a given constant field $B_{0}$ is estimated as shown in diagrams in Fig. 12(a) ([16]). At given constant field $B_{0}$ (here $0.4 \mathrm{~T}$ ) and a given strain $\gamma$, the MRE $E_{\mathrm{mag}}$ is given by:

$$
\begin{aligned}
E_{\text {mag }}=2\left[\int_{0}^{H_{0}}\right. & B(\gamma \neq 0) d H \\
& \left.-\int_{0}^{H_{0}} B(\gamma=0) d H\right] \\
& =\frac{2}{\mu_{0}} \int_{0}^{B_{0}} \Delta B(\gamma, B) d B_{0}
\end{aligned}
$$

the factor of 2 arising from the fact that as $\gamma$ is cycling between $+\gamma_{0}$ to $-\gamma_{0}$ over one period, so the $\Delta B$ curve is symmetric with the strain as seen in Fig. 11. Since the application of shear is 
reducing the induction (Fig. 12(a)), $\Delta B$ is negative and the change of magnetic density energy $E_{\text {mag }}$ due to the shear is also negative.
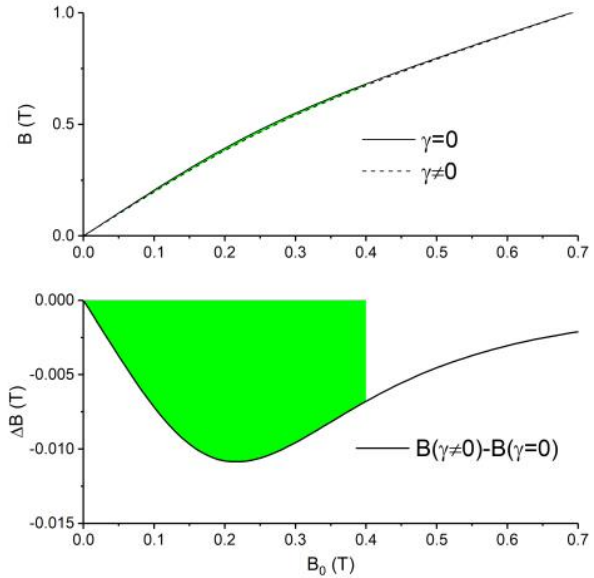

(a)

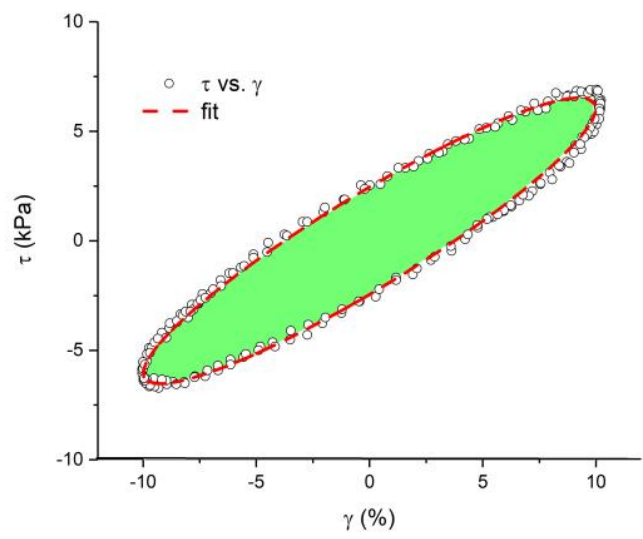

(b)

Fig.12. a) Schematic curves of $B(\gamma=0)$ and $B(\gamma \neq 0)$ versus applied $B_{0}$ (top) and corresponding $\Delta B$ (bottom); the area colored in green corresponds to $E_{\mathrm{mag}}$ for field applied up to 0.4 T. b) Stress-strain curves of MRE; the area colored in green corresponds to $E_{\text {mech. }}$

Such results were calculated for each MRE under different strain amplitudes and bias fields. Results are presented in Fig. 13(a) as a function of the shear strain amplitude $\gamma_{0}$ for each MRE (corresponding to a filling factor $\phi$ ). For a given MRE, increasing shear strain $\gamma_{0}$, increases $E_{\text {mag }}$ (in absolute value) as well. Similar to the shear strain effect, the field also enhances $E_{\text {mag }}$ because the integration upper limit in equation (12) is larger as seen in corresponding diagram of Fig. 12(a). However, the increase rate becomes smaller; for example, for MRE15 with $\gamma_{0}=30 \%$, the energy density values yield $E_{\mathrm{mag}}$ $-160,-470,-770,-980,-1125-1210$ and $1240 \mathrm{~J} / \mathrm{m}^{3}$ for $B_{0}=0.1$, $0.2,0.3,0.4,0.5,0.6$ and $0.7 \mathrm{~T}$ respectively. $E_{\mathrm{mag}}$ also increases with the filling factor, but this increase is getting lower when $\phi$ is larger than $15 \%$, as highlighted by the dashed line plot.

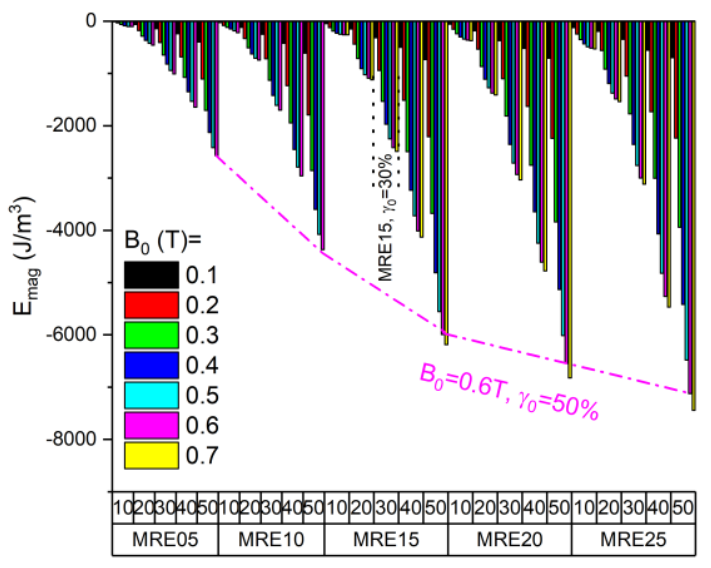

(a)

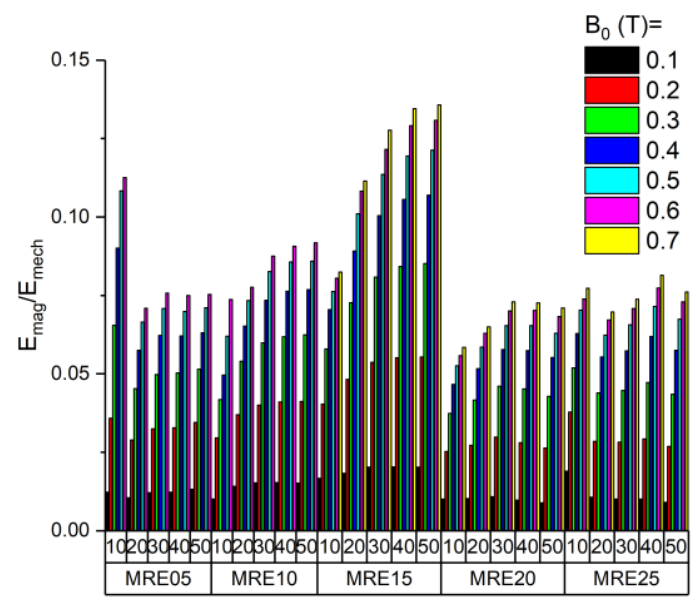

(b)

Fig.13. a) Magnetic density energy converted due to the shear as defined in equation (13) for different the filling factors, applied shear strain amplitudes $\gamma_{0}$ and applied field $B_{0}$; b) Ratio between this magnetic energy and the mechanical energy.

The input mechanical density energy is the loop area in the stress-strain curves shown in Fig. 12(b), estimated over one period as:

$$
E_{\text {mech }}=\int_{0}^{1 s} \tau \frac{\partial \gamma}{\partial t} d t
$$

The ratio of the output magnetic density energy (equation (13)) by the mechanical energy (equation (14)) used to shear the MRE under different strain amplitudes and applied fields is presented in Fig. 13(b). It can be seen that as the amplitude of shear strain $\gamma_{0}$ increases, this ratio is enhanced. In Fig.10, 
an optimal value of field $B_{0}$ was reported for the voltage amplitude $V \mathrm{~s}$ and the extracted change of flux density $\Delta B$ (Fig.11) is also marked by the same optimal value of applied field. These optima were found to be around the saturating field (Table 3) which also follow the same trend with increasing the filling factor. This optimal value was, to some extent, dependent on the amplitude of shear strain too. In the case of the ratio between the magnetic energy and the mechanical energy, Fig.13(b), for MRE05 $(\phi=5 \%)$ at given $\gamma_{0}$, optimal values were increasing from $0.1 \mathrm{~T}$ for $\gamma_{0}=10 \%$ to $0.3 \mathrm{~T}$ for $\gamma_{0}=50 \%$. An interesting result is the good performance obtained for MRE15 $(\phi=15 \%)$ at $\gamma_{0}=50 \%$ and applied field of $0.7 \mathrm{~T}$. As already mentioned, the largest filling factor does not lead to an improvement of the output voltage (Fig.10), so that increasing the filling factor does not seem to be a good solution to increase this ratio. The fact that the efficiency ratio is lower for $\phi=20 \%$ or $25 \%$ can be due to the strong mechanical energy increase with larger filling factor (equation (14)) as seen in Figs. 5-7.

\section{Conclusion}

In this paper, we present a deep parametric analysis of the output voltage and conversion efficiency of a device converting mechanical shear oscillation into electrical signal. This device uses two anisotropic Magneto Rheological Elastomers, which were placed into a magnetic circuit and submitted to shear strain. This causes a change of flux in the magnetic circuit which is then converted into output voltage. The output voltage depends then on: (i) the filling factor, (ii) the shear amplitude and (iii) the applied constant field. While the results revealed that voltage amplitude increases with applied shear, the effect of filling factor and applied bias field were less straightforward. It was found that the increase of filling factor over $15 \%$ did not improve the voltage so much and a maximum voltage was found at a specific optimal applied field $B_{\text {opt }}$. Best condition for the electric voltage amplitude was found using the MRE15, MRE20 or MRE25 with $B_{\text {opt }}=0.2 \mathrm{~T}$ and applied shear amplitude of for $\gamma_{0}=50 \%$.

From the magneto-mechanical measurements, we demonstrated that the magnetic particles are saturated at relatively low saturating field $H_{\text {sat_p }}$, because of their columnar structure, and this field $H_{\text {sat_p }}$ and $B_{\text {opt }}$ were relatively close to each other.

As both the filling factor and applied constant field seem to have a limited impact on $\Delta B$, the best way to improve $\Delta B$ is to increase the applied shear strain deformation. However, this was also predicted to have some limit because of the drastic reduction of magnetic interaction between particles, when inter-particle distance increases [18]. Other architectures might improve the effect and will be the subject of further works.

\section{Acknowledgements}

M. Lallart gratefully acknowledges the support of JSPS through invitational fellowship grant number L19530, as well as INSA-Lyon for its support through the CRCT program.

The project is supported by French Agence Nationale de la Recherche, Investissements d'Avenir, Project IDEXLyon of the Université de Lyon [grant number ANR-16-IDEX-0005].

\section{References}

[1] Li Y, Li J, Li W and Du H 2014 Smart Mater. Struct. 23123001

[2] Asun Cantera M, Behrooz M, Gibson RF and Gordaninejad F 2017 Smart Mater. Struct. 26023001

[3] Jolly MR, Carlson JD and Muñoz BC 1996 Smart Mater. Struct. 5607

[4] Kim YK, Koo JH, Kim KS and Kim SH 2011 Rev. Sci. Instrum. 82035103

[5] JYang J, Sun S, Tian T, Li W, Du H, Alici G and Nakano M 2016 Mechanical Systems and Signal Processing 70811

[6] Diguet G, Beaugnon E and Cavaillé JY 2009 J. Magn. Magn. Mater. 321396

[7] Diguet G, Beaugnon E and Cavaillé JY 2010 J. Magn. Magn. Mater. 3223337

[8] Danas K 2017 J. Mech. Phys. Sol. 10525

[9] Varga Z, Filipcsei G and Zrinyi M 2006 Polymer 47227

[10] Martin JE, Venturini E, Odinek J and Anderson RA 2000 Phys. Rev. E $\mathbf{6 1} 2818$

[11] Zhou GY and Jiang ZY 2004, Smart Mater. Struct. 13309

[12] Kushch VI and Sevostianov I 2014 Int. J. Eng. Sci. 7415

[13] Iwamoto Y, Kondoh S, Ido Y, Yamamoto H, Nishida H, Yamasaki H, Yamaguchi H and Jeyadevan B 2018 Int. J. Appl. Electromagn. Mech. 58371

[14] Tu JW, Yu Y, Huang L, Tu B and Xu JY 2014 Mater. Resear. Inno. 18243

[15] Lanotte L, Ausanio G, Hison C, Iannotti V, Luponio C and Luponio C Jr. 2004 J. Optoelectr. Adv. Mater. 6523

[16] Sebald G, Nakano M, Lallart M, Tian T, Diguet G and Cavaille JY 2017 Sci. Tech. Adv. Mater. 18766

[17] Lallart M, Sebald G, Diguet G, Cavaille JY and Nakano M 2017 J. Appl. Phys. 122103902

[18] Diguet G, Sebald G, Nakano M, Lallart M and Cavaille JY 2019 J. Magn. Magn. Mater. 48139

[19] Garnett JCM 1904 Phil. Trans. Roy. Soc. A, 203385

[20] Martin JE, Venturini E, Odinek J and Anderson RA 2000 Phys. Rev. E 612818

[21] Boczkowska A, Awietjan SF and Wroblewski R 2007 Smart Mater. Struct. 161924

[22] Boczkowska A, Awietjan SF, Wroblewski R and Kurzydłowski KJ 2009 J Mater Sci 443135

[23] Jalocha D, thesis manuscript 2015.

[24] Guth E 1945 J. Appl. Phys. 1620

[25] Guth E and Gold O 1938 Phys. Rev. E 53322

[26] Flandin L, Hiltner A and Baer E 2001 Polymer 42827

[27] Sato M and Ishii Y 1989 J. Appl. Phys. 66983

[28] Gunther D, Borin DY, Gunther S and Odenbach S 2012 Smart Mater. Struct. 21015005.

[29] Chen L, Gong XL and Li W, 2007 Smart Mater. Struct. 16 2645 